\title{
Isomer ratios for products of photonuclear reactions on ${ }^{121} \mathrm{Sb}$
}

\author{
Oleg Bezshyyko $^{1}$, Anatoliy Dovbnya ${ }^{2}$, Larisa Golinka-Bezshyyko ${ }^{1, \text { a }}$, Igor Kadenko ${ }^{1}$, Oleksandr Vodin ${ }^{2}$, Stanislav \\ Olejnik $^{2}$, Gleb Tuller ${ }^{2}$, Volodymyr Kushnir ${ }^{3}$, and Viktor Mitrochenko ${ }^{3}$ \\ 1 NPD, Taras Shevchenko National University, Kyiv, Ukraine \\ 2 National Science Center "Kharkiv Institute of Physics \& Technology" (NSC KIPT), Kharkiv, Ukraine \\ 3 Research and Development Complex "Accelerator" of NSC KIPT, Kharkiv, Ukraine
}

\begin{abstract}
Over the past several years various preequilibrium model approaches for nuclear reactions were developed. Diversified detailed experimental data in the medium excitation energy region for nucleus are needed for reasonable selection among these theoretical models. Lack of experimental data in this energy region does essentially limit the possibilities for analysis and comparison of different preequilibrium theoretical models. For photonuclear reactions this energy region extends between bremsstrahlung energies nearly $30-100 \mathrm{MeV}$. Experimental measurements and estimations of isomer ratios for products of photonuclear reactions with multiple particle escape on antimony have been performed using bremsstrahlung with end-point energies 38, 43 and $53 \mathrm{MeV}$. Method of induced activity measurement was applied. For acquisition of gamma spectra we used HPGe spectrometer with $20 \%$ efficiency and energy resolution $1.9 \mathrm{keV}$ for $1332 \mathrm{keV}$ gamma line of ${ }^{60} \mathrm{Co}$. Linear accelerator of electrons LU-40 was a source of bremsstrahlung. Energy resolution of electron beam was about $1 \%$ and mean current was within (3.8-5.3) $\mu$ A.
\end{abstract}

\section{Introduction}

Using high energy gamma-quanta as projectiles in nuclear reactions has some essential advantages to study of nuclear structure and nuclear reaction mechanisms. Indeed, gamma-quanta do not introduce large angular momentum into compound nucleus and additional contribution to excitation energy of compound nucleus due to binding energy of projectile is absent. In addition, the precise nondiscrete control of the gamma-quanta energy is possible.

Characteristics of photonuclear reactions are well studied in the energy region of Giant Dipole Resonance (GDR) and above the pion-producing threshold (PPT). The energy region between GDR and PPT (from about 30 to about $100 \mathrm{MeV}$ ) was studied to a smaller extent both theoretically and experimentally. The reason is due to small values of photonuclear reaction cross sections in this energy region and limited availability of high intensity quasi mono-energetic gamma ray sources with well controlled gamma-quanta energy.

During the last several years essential progress has been achieved in development of the new theoretical models for the photonuclear reactions and in improvement of the existing ones in the considered energy region. The quasi-deuteron model was further improved [1], some new pre-equilibrium models have been developed for description of the multi-particle emission [2]. Permanently growing interest in Accelerator Driven Systems and progress in the design of high intensity quasi monoenergetic gamma-quanta sources [5] also stimulates study of the photonuclear reactions above the GDR energy region. Very limited experimental data for the

a e-mail: lyalkagb@gmail.com photonuclear reactions in the energy range (30-100) MeV for testing newly developed and available theoretical models was the major motivation for the present work.

The main purpose of this study is to obtain the experimental isomer ratios for nuclei ${ }^{118 m, g} \mathrm{Sb}$, ${ }^{116 m, g} \mathrm{Sb}$ as the products of the ${ }^{121} \mathrm{Sb}(\gamma, 3 \mathrm{n})^{118 m, g} \mathrm{Sb}$ and ${ }^{121} \mathrm{Sb}(\gamma, 5 \mathrm{n}){ }^{116 m, g} \mathrm{Sb}$ reactions.

\section{Methodology}

De-excitation time of nuclei by the $\gamma$-cascade irradiation usually does not exceed $10^{-12} \mathrm{~s}$ [4]. In some cases transitions between levels of nucleus are suppressed due to the large difference of angular momentum of these levels involved and the nucleus can live long enough in a specific state called the isomer state. Usually this isomer state doesn't have large excitation energies and its angular momentum differs from a spin of the ground state by a few units of $\hbar$.

The isomer or ground levels with large values of spin are populated mainly from highly excited states with large spin values. Population of isomer or ground levels with smaller values of spin can occur mainly from highly excited states with small values of spins. Therefore investigations of relative populations of the isomer and ground states $[5,6]$ can be very useful to derive spins of highly excited levels and to study the de-excitation mechanisms via gamma emission.

For mono-energetic gamma beam with energy $\mathrm{E}$ the isomer ratio is determined as the cross sections ratio $\sigma_{m}(E) / \sigma_{g}(E)$, where $\sigma_{g}(E)$ is the cross section of the photonuclear reaction leading to the ground state, $\sigma_{m}(E)$ is the cross section for the same nucleus leading to the isomer state. Also the isomer ratio is often determined as a ratio of 
the cross section $\sigma_{H}$ for state with higher spin to the cross section $\sigma_{L}$ for state with lower spin:

$$
\xi=\sigma_{H} / \sigma_{L}
$$

If a gamma beam is non-monoenergetic (this is the case for experiments with bremsstrahlung sources), the isomeric yield ratio is then being determined as:

$$
d\left(E_{\max }\right)=Y_{m} / Y_{g}
$$

where the reaction yield is given by expression

$$
Y_{m, g}=N_{t} \int_{E_{t h r}^{m, g}}^{E_{\max }} \sigma_{m, g}(E) \cdot W\left(E, E_{\max }\right) d E
$$

$N_{t}$ - number of the target nuclei, $Y_{m, g}$ - reaction yield for nucleus in the isomer $(m)$ or ground $(g)$ state, $E_{\text {max }}$ maximal gamma energy, $W\left(E, E_{\text {max }}\right)$ - bremsstrahlung spectrum, $\sigma_{i}(E)$ with $i=m, g-$ the reaction cross section for nucleus to be formed in meta-stable (ground) state for gamma energy $E, E_{t h r}^{m, g}, i=m, g-$ the energy threshold of the reaction leading to the meta-stable (ground) state.

The production of isomeric pair and its decay can be described by the following differential equation system:

$$
\left\{\begin{array}{l}
\frac{d N_{m}}{d t}=Y_{m}-\lambda_{m} \cdot N_{m} \\
\frac{d N_{g}}{d t}=Y_{g}-\lambda_{g} \cdot N_{g}+p \cdot \lambda_{m} \cdot N_{m}
\end{array},\right.
$$

where $N_{i}$ - population of i-state $(i=m$ - isomer state, $i=g-$ ground state), $Y_{i}-$ reaction yield according to (3), $\lambda_{m}, \lambda_{g}$ - decay constants for isomer and ground state, $\mathrm{p}$ - branching factor (transition probability from isomer to ground state) $[7,8]$. The equation system is valid under such conditions: gamma-quanta flux is time invariable; contribution from interfering reaction may be considered as negligible; simple decay scheme, when the isomer level decays by gamma transition to the ground state in competition with $\beta$-decay and the ground state decays by $\beta$-decay branch.

Solution of system (2) is:

$$
\left\{\begin{array}{l}
\frac{S_{m}}{C_{m} \varepsilon_{m} f_{m}}=Y_{m} \Lambda_{3} \Lambda_{6} \Lambda_{9} \\
\frac{S_{g}}{C_{g} \varepsilon_{g} f_{g}}=Y_{g} \Lambda_{2} \Lambda_{5} \Lambda_{8}+ \\
\quad+Y_{m}\left(\Lambda_{1} \Lambda_{5} \Lambda_{8}+\Lambda_{3} \Lambda_{4} \Lambda_{8}+\Lambda_{3} \Lambda_{6} \Lambda_{7}\right)
\end{array}\right.
$$

where $S_{i}, i=g, m$ - photo-peak area (in the gamma spectrum of the activation products), coefficient $\mathrm{C}$ includes self-absorption factor, true coincidence effects of cascade gammas and other effects, $\varepsilon$ - full efficiency of gamma detection for the analysed gamma-line; $f_{i}, i=g, m-$ quantum yield of gamma-line for i-state decay (transition probability for this line); coefficients $\Lambda_{j}, j=1,9$ are defined by $t_{1}, t_{2}, t_{3}$, - irradiation time, cooling time and measurement time, respectively:

$$
\begin{aligned}
& \Lambda_{1}=\frac{p}{\lambda_{g}}\left[1-\frac{\lambda_{m} \lambda_{g}}{\lambda_{m}-\lambda_{g}}\left(\frac{e^{-\lambda_{g} t_{1}}}{\lambda_{g}}-\frac{e^{-\lambda_{m} t_{1}}}{\lambda_{m}}\right)\right] \\
& \Lambda_{2}=\frac{1}{\lambda_{g}}\left(1-e^{-\lambda_{g} t_{1}}\right), \quad \Lambda_{3}=\frac{1}{\lambda_{m}}\left(1-e^{-\lambda_{m} t_{1}}\right) \\
& \Lambda_{4}=\frac{p \cdot \lambda_{m}}{\left(\lambda_{m}-\lambda_{g}\right)}\left(e^{-\lambda_{g} t_{2}}-e^{-\lambda_{m} t_{2}}\right) \\
& \Lambda_{5}=e^{-\lambda_{g} t_{2}}, \quad \Lambda_{6}=e^{-\lambda_{m} t_{2}} \\
& \Lambda_{7}=\frac{p \cdot \lambda_{m}}{\left(\lambda_{m}-\lambda_{g}\right)}\left(e^{-\lambda_{g} t_{3}}-e^{-\lambda_{m} t_{3}}\right) \\
& \Lambda_{8}=1-e^{-\lambda_{g} t_{3}}, \quad \Lambda_{9}=1-e^{-\lambda_{m} t_{3}}
\end{aligned}
$$

As a result, the following expression is obtained

$$
F=Y_{m}^{\prime} X+Y_{g}^{\prime}
$$

where $F$ and $X$ are defined as

$$
\begin{aligned}
& F=\frac{S}{\varepsilon f_{g} \Lambda_{2} \Lambda_{5} \Lambda_{8}} \\
& X=\frac{\left(\Lambda_{1} \Lambda_{5} \Lambda_{8}+\Lambda_{3} \Lambda_{4} \Lambda_{8}+\Lambda_{3} \Lambda_{6} \Lambda_{7}\right)+\frac{f_{m}}{f_{g}} \Lambda_{3} \Lambda_{6} \Lambda_{9}}{\Lambda_{2} \Lambda_{5} \Lambda_{8}}
\end{aligned}
$$

with $S=S_{g}+S_{m}$ peak area sum, $Y_{m, g}^{\prime}=C Y_{m, g}$ - values, proportional to reaction yields.

In this particular case the isomer yield ratio was calculated by fitting the experimental data $(X, F)$ using Eq. (6).

Experimentally, the method of induced activity was applied to obtain the isomer ratios. Similar approach already was used by our group on some target nuclei and showed a good result [9-12]. Irradiations of Sb target have been carried out with bremsstrahlung endpoint energies within the region (38-53) MeV. Linear accelerator LU40 (Research and Development Complex "Accelerator" NSC KIPT) was used as a source of fast electrons [13]. Instability of electron beam intensity was within $2 \%$. Inner monitor of electron beam was calibrated by values from Faraday cup of the magnetic analyzer, placed at the accelerator outlet. The tantalum converter with $1.05 \mathrm{~mm}$ thickness was placed on the exit window of the accelerator facility, close to which the cylindrical aluminium gamma absorbers (thickness 5.5 and $10 \mathrm{~cm}$ ) were installed. Diameter of beam spot on the conversion target was less than $9 \mathrm{~mm}$. Energy of electron beam was determined using magnetic analyzer and was double checked in the low energy region by considering the photonuclear reaction thresholds. A distance between tantalum converter and absorber was $2 \mathrm{~cm}$, between tantalum converter and target $-20 \mathrm{~cm}$. We used metallic antimony target with natural isotopic abundance to study the reaction ${ }^{121} \mathrm{Sb}(\gamma, 3 \mathrm{n}){ }^{118 m, g} \mathrm{Sb}$ and the reaction ${ }^{121} \mathrm{Sb}(\gamma, 5 \mathrm{n}){ }^{116 m, g} \mathrm{Sb}$. Irradiation time for every sample was $5 \mathrm{~min}$. Then within (3-8) seconds the irradiated sample was moved with pneumatic transfer system to the measurement area. HPGe detector with the energy resolution $<2.0 \mathrm{keV}$ for ${ }^{60} \mathrm{Co}$ $\gamma$-line $1332 \mathrm{keV}$ was used to acquire the instrumental gamma-ray spectra of the activation products as a set of serial measurements with various time periods. Cooling times varied from 5 seconds to few hours. Distances 


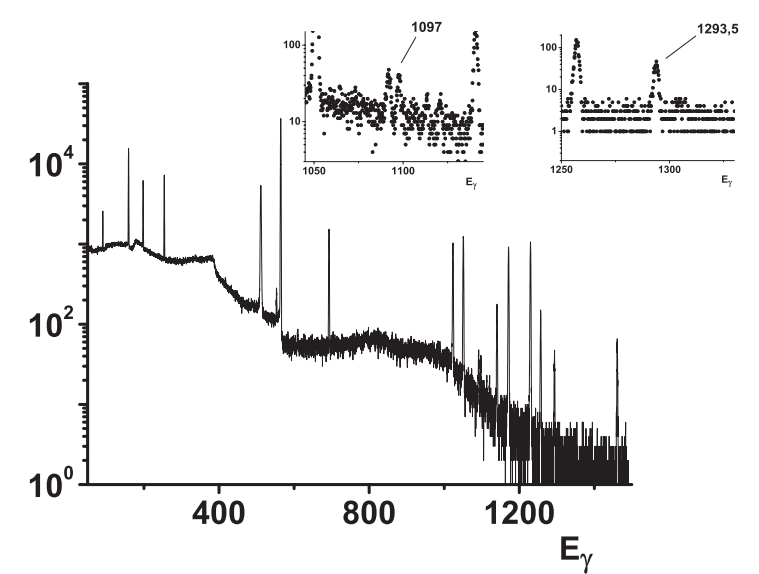

Figure 1. Gamma-ray spectra from the antimony activities. The analytical gamma lines used for determination of the isomer ratios are indicated separately.

between sample and detector (dozens centimetres just after irradiation and few centimetres at the end of measurement period) were chosen to optimize both statistics and time restrictions when large contribution of interfering reactions took place, minimum distance was restricted by condition of negligible contribution of cascade gammas summing. Efficiency calibration of spectrometer was carried out for each detector-to-sample distance. The efficiency-energy dependence in double logarithmic scale showed a good quality and linearity in the energy range of interest, with deviations between experimental data and linear fitted values not exceeding $2 \%$.

\section{Results and discussion}

The isomer ratios were obtained as $I R\left(E_{\gamma}\right)=\frac{Y_{H}\left(E_{\gamma}\right)}{Y_{L}\left(E_{\gamma}\right)}$, where $Y_{H}\left(E_{\gamma}\right)$ is the reaction yield for the state of final nucleus with larger angular momentum (meta-stable state), $Y_{L}\left(E_{\gamma}\right)$ is the reaction yield for the state of final nucleus with smaller angular momentum (ground state). Gamma transitions and corresponding lines with the energies $1050.7 \mathrm{keV}\left(\mathrm{EC}+\beta^{+}\right.$decay of the isomer state) and $1229.7 \mathrm{keV}$ (common line for decay of the ground and isomer states) were used to calculate the isomer ratio for ${ }^{118 m, g} \mathrm{Sb}$ (reaction ${ }^{121} \mathrm{Sb}(\gamma, 3 \mathrm{n}){ }^{118 m, g} \mathrm{Sb}$ ).

We used the decay scheme of the ${ }^{116 m, g} \mathrm{Sb}$ and ${ }^{118 m, g} \mathrm{Sb}$ nuclei from [4].

The examples of spectra from the induced activities for the antimony target are shown in Fig. 1. The gamma line $1293.5 \mathrm{keV}$ (common line $\mathrm{EC}+\beta^{+}$decay of the ground and isomer state) was used to obtain the isomer ratio for ${ }^{116 \mathrm{~m}, g} \mathrm{Sb}$ (reaction ${ }^{121} \mathrm{Sb}(\gamma, 5 \mathrm{n}){ }^{116 \mathrm{~m}, g} \mathrm{Sb}$ ). The gamma line $1097 \mathrm{keV}$ ( $\gamma$-decay of the isomer state) was used to account contribution of $116 \mathrm{~m}, \mathrm{~g}$ In (reaction $\left.{ }^{121} \mathrm{Sb}(\gamma, \mathrm{n} \alpha)^{116 m, g} \mathrm{In}\right)$.

One can see (Fig. 2) the fitting result of the decay line in presentation (X, F) according to Eqs. (6), (7).

All obtained experimental values of the isomer ratios, corresponding reactions and characteristics of investigated nuclei are presented in Table 1.

The uncertainties given in Table 1 include contributions from photopeak efficiency calibration, abundance, geometry configuration and intensities of gamma-rays (photopeak areas). Statistical uncertainties of photopeak

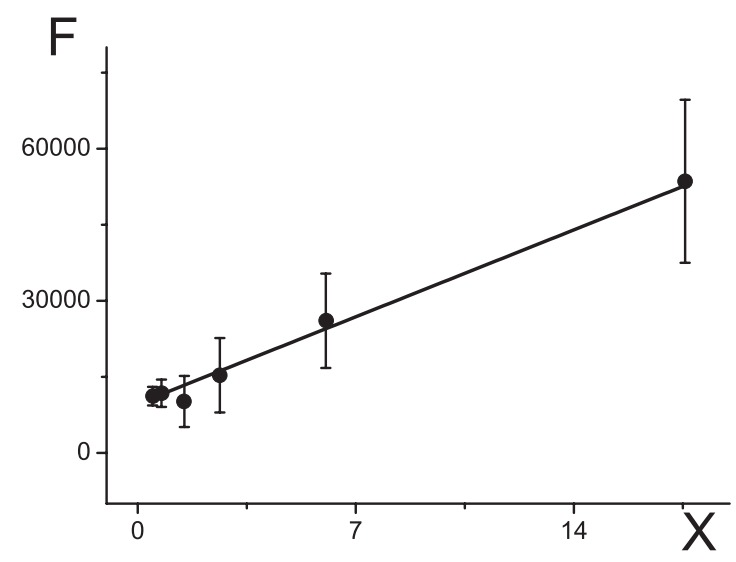

Figure 2. Fitting line for experimental points $(\mathrm{X}, \mathrm{F})$ of antimony ${ }^{116 \mathrm{~m}, \mathrm{~g}} \mathrm{Sb}$ nuclei decay (reaction $\left.{ }^{121} \mathrm{Sb}(\gamma, 5 \mathrm{n}){ }^{116 \mathrm{~m}, \mathrm{~g}} \mathrm{Sb}\right)$.

Table 1. Reactions, the bremsstrahlung energy end-points $E \gamma_{\max }$, spins of the target nuclei $\left(I_{t}\right)$, spins of the meta-stable $\left(I_{m}\right)$ and ground $\left(I_{g}\right)$ states and the experimental isomer ratios $I R=Y_{H} / Y_{L}$, obtained in experiments.

\begin{tabular}{|l|c|c|c|c|c|}
\hline Reaction & $\begin{array}{c}E_{\gamma \max }, \\
M e V\end{array}$ & $I_{t}$ & $I_{m}$ & $I_{g}$ & $I R$ \\
\hline${ }^{121} \mathrm{Sb}(\gamma, 3 \mathrm{n})^{118 \mathrm{~m}, \mathrm{~g}} \mathrm{Sb}$ & 38 & $5 / 2^{+}$ & $8^{-}$ & $1^{+}$ & $0.14 \pm 0.04$ \\
\cline { 2 - 6 } & 43 & $5 / 2^{+}$ & $8^{-}$ & $1^{+}$ & $0.15 \pm 0.01$ \\
\hline${ }^{121} \mathrm{Sb}(\gamma, 5 \mathrm{n})^{116 \mathrm{~m}, \mathrm{~g}} \mathrm{Sb}$ & 53 & $5 / 2^{+}$ & $8^{-}$ & $3^{+}$ & $0.25 \pm 0.03$ \\
\hline
\end{tabular}

Table 2. Calculations of isomer ratios using code TALYS with various model approaches: LD1 - Constant temperature + Fermi gas model; LD2 - Back-shifted Fermi gas model; LD3 Generalised superfluid model; LD4 - Microscopic level densities (Skyrme force) from Goriely's tables; LD5 - Microscopic level densities (Skyrme force) from Hilaire's combinatorial tables; LD6 - Microscopic level densities (temperature dependent HFB; Gogny force) from Hilaire's combinatorial tables.

\begin{tabular}{|l|l|l|l|l|l|l|l|}
\hline Reaction & $\begin{array}{l}E_{\gamma m} \\
M e V\end{array}$ & $L D 1$ & $L D 2$ & $L D 3$ & $L D 4$ & $L D 5$ & $L D 6$ \\
\hline $\begin{array}{l}{ }^{121} \mathrm{Sb}(\gamma, 3 \mathrm{n}) \\
{ }^{118 \mathrm{~m}, \mathrm{~g}} \mathrm{Sb}\end{array}$ & 38 & 0.061 & 0.069 & 0.063 & 0.080 & 0.098 & 0.103 \\
\cline { 2 - 8 } & 43 & 0.085 & 0.093 & 0.111 & 0.111 & 0.133 & 0.141 \\
\hline${ }^{121} \mathrm{Sb}(\gamma, 5 \mathrm{n})$ & 53 & 0.155 & 0.153 & 0.147 & 0.197 & 0.261 & 0.119 \\
\hline
\end{tabular}

areas made the main contribution to total uncertainty of result. Also number of experimental points of $F, X$ dependency and range of $X$ determination influenced total experimental error. These values were limited by experimental conditions.

The irradiation energy is very close to threshold of interfering reaction ${ }^{123} \mathrm{Sb}(\mathrm{g}, 5 \mathrm{n})^{118 \mathrm{~m}, \mathrm{~g}} \mathrm{Sb}$ and its cross section is smaller than 0.01 part of studied cross section. This contribution was taken into account, using Talys code.

We used code TALYS 1.8 [14] for theoretical calculations of isomer ratios for investigated nuclei (see Table 2).

\section{Conclusion}

The experimental values of the isomer ratios are obtained for ${ }^{118 m, g} \mathrm{Sb}$ and ${ }^{116 \mathrm{~m}, \mathrm{~g}} \mathrm{Sb}$ nuclei as products of the photonuclear reactions ${ }^{121} \mathrm{Sb}(\gamma, 3 \mathrm{n}){ }^{118 \mathrm{~m}, \mathrm{~g}} \mathrm{Sb}$ and 
${ }^{121} \mathrm{Sb}(\gamma, 5 \mathrm{n}){ }^{116 \mathrm{~m}, \mathrm{~g}} \mathrm{Sb}$, using bremsstrahlung endpoint energies in the region from 33 to $53 \mathrm{MeV}$. To correctly derive the isomer ratio for ${ }^{116 \mathrm{~m}, \mathrm{~g}} \mathrm{Sb}$ we took into account the contribution of ${ }^{116 \mathrm{~m}, \mathrm{~g}} \mathrm{In}$ from reaction ${ }^{121} \mathrm{Sb}(\gamma, \mathrm{n} \alpha)^{116 \mathrm{~m}, \mathrm{~g}} \mathrm{In}$.

Experimental values of isomer ratios for ${ }^{118 \mathrm{~m}, \mathrm{~g}} \mathrm{Sb}$ from the reaction ${ }^{121} \mathrm{Sb}(\gamma, 3 \mathrm{n}){ }^{118 \mathrm{~m}, \mathrm{~g}} \mathrm{Sb}$ and isomer ratios for ${ }^{116 \mathrm{~m}, \mathrm{~g}} \mathrm{Sb}$ from the reaction ${ }^{121} \mathrm{Sb}(\gamma, 5 \mathrm{n}){ }^{116 \mathrm{~m}, \mathrm{~g}} \mathrm{Sb}$ are in a sufficiently good agreement with theoretical calculation results, especially for LD6 end LD5 models.

The research was conducted in the scope of the IDEATE International Associated Laboratory (LIA).

\section{References}

[1] M.B. Chadwick, P. Oblozinsky, P.E. Hodgson, G. Reffo, Phys. Rev. C 44, 814 (1991)

[2] P. Talou, T. Kawano, P.G. Young and M.B. Chadwick, Nucl. Instrum. Meth. A 562, 823 (2006)

[3] http://higs.tunl.duke.edu

[4] http://www.nndc.bnl.gov/ensdf

[5] B.C. Ishkhanov, I.M. Kapitonov, Interaction of electromagnetic radiation with nuclei. M MSU. 215 (1979)
[6] Yu.P. Gangrsky, A.P. Tonchev, N.P. Balabanov, PEPAN 27, 1043 (1996)

[7] D. Kolev, E. Dobreva, N. Nenov, V. Todorov Nucl. Instr. and Meth. in Phys. Res. A 356, 390 (1995)

[8] O.A. Bezshyyko, A.N. Vodin, L.O. Golinka-Bezshyyko et al. 2nd Int. Conf. Proc. "NPAE 2008" (Kyiv Ukraine) 2, 252 (2008)

[9] O.A. Bezshyyko, A.N. Vodin, L.O. Golinka-Bezshyyko et al. Nucl. Phys. and Atomic Energy 10(1), 61 (2009)

[10] O.A. Bezshyyko, A.N. Vodin, L.A. GolinkaBezshyyko et al. Bulletin of the Russian Academy of Sciences: Physics 73(11), 1461 (2009)

[11] O.A. Bezshyyko, A.N. Vodin, L.A. Golinka-Bezshyyko et al. Bulletin of the Russian Academy of Sciences: Physics 75(4), 937 (2011)

[12] O.A. Bezshyyko, A.N. Vodin, L.O. Golinka-Bezshyyko et al. Bulletin of the Russian Academy of Sciences: Physics 75(4), 941 (2011)

[13] A.N. Dovbnya, M.I. Aizatsky, V.N. Boriskin, I.V. Khodak, V.A. Kusnir, V.V. Mytrochenko, A.N. Opanasenko, S.A. Perezhogin et al. Prob. of Atom. Sci. \& Tech. 2, 11 (2006)

[14] http://www.talys.eu/ 